\title{
An Insight into Relationship of Hypertrophied Adenoids \& Tonsils and Dentofacial Form
}

\author{
Ravneet Kaur ${ }^{1}$, Chandrashekhar Gandikota ${ }^{2}$, Dhawal R. Mody ${ }^{3}$, Poornima KY ${ }^{4}$, \\ Shubhaker Rao Juvvadi ${ }^{5}$ \\ ${ }^{1}$ Senior lecturer, Dept. of Orthodontics, Panineeya Dental College \& Hospital, Andhra Pradesh, India \\ ${ }^{2}$ Prof. \& Head, Dept. of Orthodontics, Panineeya Dental College \& Hospital, Andhra Pradesh, India \\ ${ }^{3}$ Senior Lecturer, Dept. of Periodontics, VSPM's Dental College \& Hospital, Nagpur, India \\ ${ }^{4}$ Professor, Dept. of Orthodontics, Panineeya Dental College \& Hospital, Andhra Pradesh, India \\ ${ }^{5}$ Reader, Dept. of Orthodontics, Panineeya Dental College \& Hospital, Andhra Pradesh, India
}

\begin{abstract}
Air Passage through nasopharynx and nose is limited by its shape and size. Nasal obstruction \& mouth breathing can be caused by multiple factors, one of them being hypertrophied adenoid and tonsils. It is perhaps one of the most widely debated reasons for partial airway obstruction and related respiratory abnormalities. Otolaryngolists are increasingly getting involved in assessment of upper airway obstructions and its effect from an orthodontic perspective. Also, surgical intervention in such cases might not be the best route given the variance of malocclusion across patients.

In this paper, we shall review some of the precursors which can serve as indicator for need of surgical and/or orthodontic intervention to reduce the dentofacial abnormalities in cases of hypertrophied adenoid and tonsils. Such precursors can also help in identification of patients' susceptibility to Obstructive sleep apnea (OSA). Further, we will review the interaction between airway problems and expressed mandibular morphology as documented in historic literature.

The article aims to bridge the communication between Orthodontist and medical fraternity by providing a lucid illustration of issues pertaining to obstructive breathing and its implication on craniofacial growth.
\end{abstract}

Key words: adenoid hypertrophy, hypertrophied tonsils, obstructive sleep apnea, orthodontics.

\section{Introduction}

Mouth breathing is one of the many factors which have a significant impact on the overall dentofacial development. While habit formation is one of the causes of mouth breathing in young children, bone anatomy of nasal track and obstructive growth like enlarged tonsils \& adenoid can also play a major role in the development of mouth breathing and its subsequent effects. While it is within the orthodontist's purview to diagnose dentofacial abnormalities, who in most cases is the first clinician to notice mouth breathing, the decision for tonsillectomy and/or adenoidectomy remains a medical decision.

The aim of this article is to provide a lucid illustration of issues pertaining to obstructive breathing and its implication on craniofacial growth to Orthodontist and medical fraternity. This review also highlights the skills and tools that assist the clinician in identifying upper airway obstruction and improves the diagnosis of adenoid hypertrophy with the treatment of associated malocclusion.

Further, recently there is a growing interest and awareness on the subject of severe upper airway obstruction due to its association with Obstructive sleep apnoea (OSA) ${ }^{1}$. The major cause of OSA in children is adenotonsillar hypertrophy and the severity of OSA is associated with the size of the hypertrophic adenoids. There is evidence that severely obstructed patients should be promptly and effectively treated with tonsillectomy. ${ }^{2}$

\section{Brief Anatomic Description Of Upper Airway}

The tonsils (palatine or faucial tonsils) and the adenoids (pharyngeal tonsils) are part of Waldeyer's tonsillar ring ${ }^{3}$ (Figure 1) (or nasal-associated lymphoid tissue (NALT), named after a nineteenth century German anatomist Heinrich Wilhelm Gottfried Waldeyer. The ring also includes the tubal tonsil, where the Eustachian tube opens in the nasopharynx and the lingual tonsils. As the tonsils and adenoids are located at the gate of the upper respiratory and alimentary tracts, where they are constantly exposed to antigens, they are mostly composed of immunologically reactive lymphoid tissue containing antibody-producing lymphocytes. 


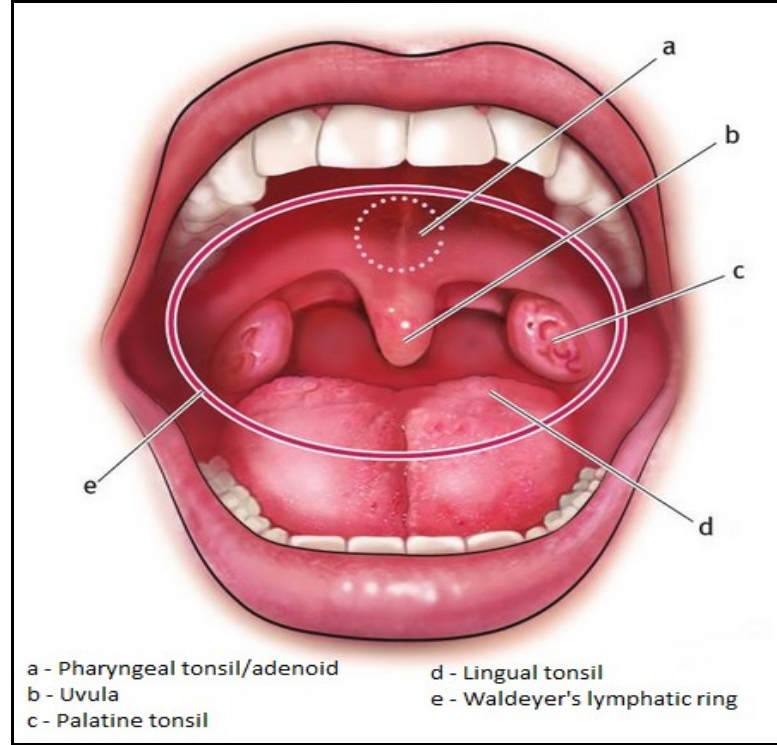

Figure 1 Waldeyer's lymphatic ring

The nasopharynx is a vault like structure that extends from the base of the skull to the level of the hard palate. The choanae forms the anterior border of the nose, whereas the pharyngeal isthmus forms the lower border, toward the oropharynx (Figure 2) ${ }^{4}$.

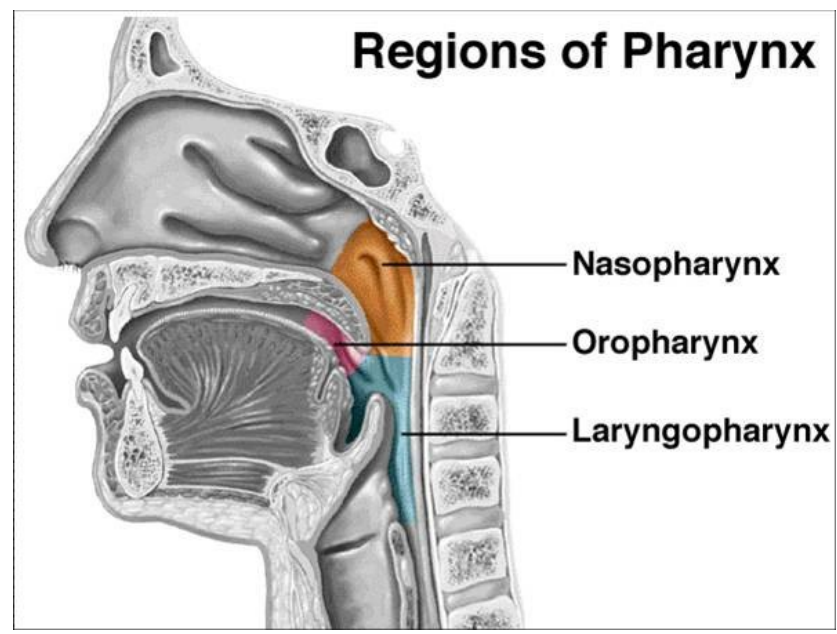

Figure 2 Anatomy of pharynx

Excessive Adenoidal growth can have severely damaging effects like abnormal facial growth together with abnormal breathing patterns, congestion, mouth breathing, sleep apnea, rhinosinusitis, swallowing problem, reduced ability to taste, smell and problems with speech. Thus it is important for the orthodontist to know the growth pattern of the posterior nasopharyngeal wall when evaluating the nasopharyngeal airway on lateral cephalogram.

Anatomically, the pharyngeal tonsil or adenoid, which appears at about the sixth month of fetal life as a subepithelial infiltration with lymphocytes,lies on the roof and upper posterior wall of the nasopharynx. ${ }^{5}$ The adenoids are small at birth due to immature state of the immune system. Development of the adenoids is unique among major tissues of the body: they increase in size to a peak of approximately $200 \%$ the adult size around puberty then decrease to near-total regression in most adults. The nasopharynx enlarges to accommodate the growing adenoids, resulting in maintenance of a patent nasopharyngeal airway but any imbalance between the developing airway and the concomitant adenoid growth may result in nasopharyngeal obstruction or reduced potency. Given their critical anatomical position, hypertrophied adenoids may affect nasal function as well as obstruct the Eustachian tubes, participating in the formation of middle ear effusions. ${ }^{4}$

The oropharyngeal space is situated dorsal to the tongue. Obstruction of the oropharyngeal space can also be caused by grossly enlarged tonsils due to chronic or recurrent acute tonsillitis. Enlarged tonsils may lead 
to a forward displacement of the tongue and a caudal displacement of the hyoid bone, changes that are reversed after a tonsillectomy.

Tonsils and/or adenoids present a health problem when they become the nest of continuous infections and cause airway obstruction. Not only does the blockage induce mouth breathing, but also a nasal twang to the voice, snoring, restless sleep (and associated learning problems if chronic), and facial dysmorphology.

\section{Mode Of Respiration And Dento-Facial Form}

Dentofacial changes associated with nasal airway blockage have been described by CV Tomes in 1872 as adenoid facies. ${ }^{6}$ Tomes coined this term based on his belief that enlarged adenoids were the principle cause of airway obstruction and resulted in noticeable dentofacial changes. Tomes reported that children, with mouth breathing, often exhibited narrow V-shaped maxillary arches. This narrow jaw is a result of mouth breathers keeping their lips apart and their tongue position low. The tongue displaced downward can lead to a retrognathic mandible and an interposed tongue can lead to anterior occlusal anomalies. Additionally, there is often presence of a narrow and retrusive maxilla concomitant with palatal inclination in relation to cranial base and an increase in lower anterior facial height. Furthermore occlusal changes like crossbite (posterior \&/or anterior), open bite, increased over jet and retroclination of maxillary and mandibular incisors may ensue.

Tomes' views were supported in the 1930's by numerous leading orthodontists. They reported airway obstruction as an important aetiologic agent in malocclusion. Rubin advocated that in order for these patients to fully be assessed they must be thoroughly evaluated by both a rhinologist and an orthodontist. ${ }^{7}$

Several authors ${ }^{8,9}$ have taken the position that adenoid faces are not consistently found to be associated with adenoids, mouth breathing, or any particular type of malocclusion and that there is no cause and effect relationship between adenoids, nasal obstruction / mouth breathing and malocclusion. Proponents of this position are of the opinion that the V-shaped palate was inherited and not acquired through mouth breathing. Hartsook on a review of literature related to mouth breathing concluded that mouth breathing is not a primary etiological factor in malocclusion.

Vig, an orthodontic clinician took a position that from an orthodontic stand point, any surgical intervention is difficult to justify without documented total nasal obstruction. ${ }^{10}$

\section{Nasopharyngeal Obstruction \& Mouth Breathing}

The factors contributing to the obstruction of upper airway include: anatomical airway constriction, developmental anomalies, enlarged tonsils and adenoids, macroglossia, nasal polyps and allergic rhinitis. ${ }^{5}$ However, this paper focuses on enlarged adenoids and tonsils as the major contributing factor. Further, upper airway obstruction resulting from enlarged adenoids and tonsils can be one of the most common causes of mouth breathing in children.

Mouth breathing resulting from blockage of nasal cavity or pharynx can sometimes result in postural modification. These postural modifications can range from open lips or a lowered tongue position, clockwise rotation of the mandible, or even a change in head posture. A malocclusion and altered skeletal relationship is likely to occur if an individual with upper airway obstruction responds in one or more of the following neuromuscular responses: 5,9

1. Altered mandibular posture: The mandible rotates down and back in response to the etiologic factor.

2. Altered tongue posture: the tongue moves superiorly and anteriorly in response to the etiologic factor

3. Extended head posture: The mandible is held in position while the cranium and maxilla rotate upward.

These modifications take place in an effort to stabilize the airway. As previously discussed, postural alterations in soft tissues produce changes in equilibrium of pressure exerted on teeth and facial bones. This could result in modification in facial structures. Additionally, during mouth breathing, alterations in the muscles affect mastication, phonation and deglutition because other muscles are relied upon. Hence, it can lead to a change in swallowing and speech pattern of an individual.

\section{Evaluation Of Nasal Respiratory Obstruction}

A thorough history and clinical examination remains the gold standard for diagnosis of all malocclusion in addition to the use of other supplemental diagnostic tools. The evaluation of nasal airway patency is complicated; more so when the possibility exists that airways may clinically appear inadequate but is quite functional physiologically. Lip incompetency or an open-mouth habit is not a perfect indicator of mouth breathing. Often complete nasal respiration is associated with dental conditions that cause open-mouth posture. Various methods of evaluation of enlargement of adenoids and upper airway patency are as follows:

A. Radiographic evaluation of adenoids

1. Four well-established measurements on lateral cephalogram from the orthodontic literature were chosen: the Holmberg and Linder-Aronson subjective method $^{11}$, McNamara's linear method ${ }^{12}$, the Kemaloglu et al. ${ }^{12}$ ratio, and the Handelman and Osborne ${ }^{14}$ area method : (fig3) 
a) Holmberg and linder-aronson method ${ }^{11}$ : the size of adenoids is graded subjectively according to the following scale: 1 = no adenoids, 2 = small adenoids, 3 = moderate adenoids, $4=$ large adenoids, 5 = very large adenoids)

b) McNamara's linear method ${ }^{12}$ : upper pharyngeal width is measured from a point on the posterior outline of soft palate to the closest point on the posterior pharyngeal wall. Apparent airway obstruction, as indicated by an opening of $5 \mathrm{~mm}$ or less in the upper pharyngeal measurement, is taken only as an indicator of possible airway impairment.

c) Ratio method (Kemaloglu) ${ }^{13}$ The adenoidal-nasopharyngeal ratio (ANR) is a convenient and practical method to evaluate adenoidal enlargement.

d) Handelman \& Osborne area method ${ }^{14}$ The trapezoidal analysis method given by Handleman and Osborne proves to be good clinical guide for measuring nasopharyngeal area on lateral cephalogram. The nasopharyngeal area was defined by four skeletally defined lines which formed a trapezoid. It was further divided into adenoid-pharyngeal wall (Ad area) and airway areas. Percentage of Ad area determines the degree of obstruction.
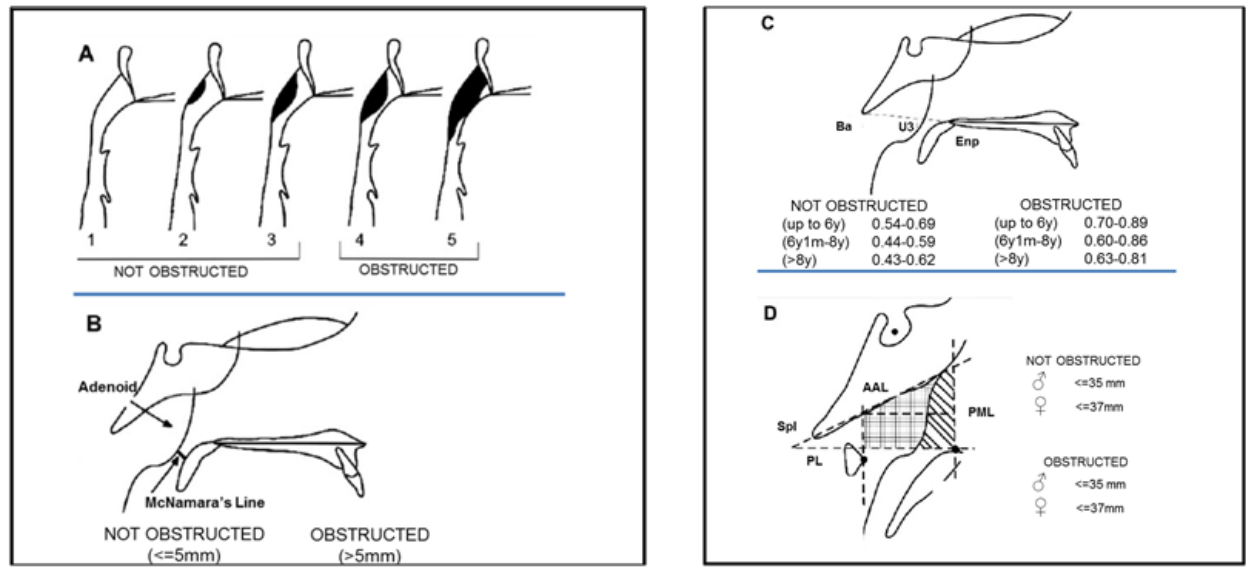

Figure 3: (A) Holmberg \& Linder-Aronson subjective method. (B) McNamara's linear method. (C) Ratio method of Kemaloglu et al (D) Handelman and Osborne area method

2. Cone Beam Computed Tomography (CBCT): The major disadvantage of using lateral cephalogram for airway analysis is that the three-dimensional (3D) structure of the airway is represented as a twodimensional (2D) image on a cephalogram. Further, detection and volumetric assessment of the exact soft tissue anatomy, is impossible because of the overlap of numerous structures. Therefore, $3 \mathrm{D}$ analysis of the airway shape and possible airway obstruction using CBCT is important. ${ }^{1}$

B. Flexible nasal endoscopy: Flexible nasal endoscopy is a quick and simple procedure to visualize the nasal passage or throat to find the cause of the symptoms.

C. Rhinomanometry: It is a standard diagnostic tool aiming to evaluate the respiratory function of the nose. It measures air flow and pressure during normal inspiration and expiration through the nose. In case of increased resistance to airflow owing to nasal blockage, the pressure during respiration increases, while increased flow, indicated by higher speed of airstream, is related to better airway patency.

D. Direct measurements during surgery

\section{Treatment Of Nasopharyngeal Obstruction}

Historically, the appearance of characteristic changes in the dentofacial form of an individual with nasal airway obstruction at an early age supported early surgical intervention to prevent its progress to fullfledged long face syndrome, However, now the pendulum has shifted to more conservative approach avoiding surgical intervention in case no postural modifications (as discussed above) are observed. Surgical intervention at an early age ( $<5$ years) to avoid irreversible morphological changes may not be advisable in general for several reasons: ${ }^{15}$

- With the advent of electrophoresis, we have learned that immunoglobulins produced by the lymphoid tissue are very important in the function of immunity. ${ }^{16}$

- The second factor that has produced the swing of the pendulum to conservatism on the part of paediatrician is a greater awareness of the allergic syndromes. It is a mistake to perform an adenoidectomy on a child with a nasal allergy, if this is done to relieve obstruction.

- If compensation occurs within the dentition (absence of crossbite, open bite, or gummy smile) and morphological changes do not get worse at a later follow-up, early surgery may not be needed 
- With increasing age, the airway is less obstructed and the effects on the palatal plane and other morphological features decrease in intensity, indicating a gradual adjustment to growth of nasopharyngeal airway and face.

Therefore, there is a need to develop guidelines for early adenoidectomy and/or tonsillectomy that account for the varied response to the obstruction and normal growth changes. Indications for early adenoidectomy might include a combination of: ${ }^{15-18}$

- Recurrent, acute, follicular tonsillitis which culminates in chronic infection \& tonsillar hypertrophy.

- quinsy i.e. peritonsillar abscess

- tuberculosis or carcinoma of tonsils

- mechanical obstruction of upper airway by adenoid or tonsils is another indication for a tonsillectomy \& adenoidectomy

- An existing skeletal dysmorphology (palatal plane tip, steep mandibular plane, or increased lower face height) probably beyond 1 SD from the norm

- Persistent nasopharyngeal airway obstruction grade 3 and grade $4 .{ }^{19}$

Various approaches towards the treatment of nasopharyngeal obstruction and its associated dentofacial features are:

\section{A. Pharmacological management:}

Pharmacological management of adenoid or tonsillar hyperplasia begins with a course of an antibiotic effective against beta-lactamase producing organisms such as clindamycin or Augmentin. Patients' resistant to above drugs can be prescribed an antibiotic effective against beta-lactamase producing organisms and anaerobes such as clindamycin, Augmentin, or penicillin plus rifampin.

\section{B. Surgical intervention:}

If hypertrophied adenoids (and tonsils) are the primary etiological factor of upper airway obstruction, adenoidectomy with or without tonsillectomy is indicated. Surgical intervention options should be chosen depending on site and degree of obstruction:

1. Adenoidectomy in conjunction with Endoscopic Visualization helps to achieve removal of adenoids particularly high in the nasopharnx. Further it also allows for better clearance of obstructive adenoids resulting in a reliable restoration of nasal patency.

2. Septal surgery, generally contraindicated in children, may be considered if there is marked nasal septal deflection with impaction. Further, conservative septal surgery in growing patients might not have an adverse effect in dentofacial growth.

3. Cryosurgery or electrosurgery - this is a viable option for patients with vasomotor rhinitis.

4. Bipolar Radiofrequency Ablation (allergic rhinitis) - performed under local anesthesia

5. Inferior turbinectomy - Using powered instrumentation

Although tonsillectomy and adenoidectomy are typical strategies and play an important role in relieving obstructive symptoms for patients with adenotonsillar hypertrophy and OSA; but these procedures are associated with serious complications such as bleeding (4-5\%)' and postoperative respiratory compromise (27\%) especially among younger children 'which should be kept in mind..$^{20,21}$

Also numerous small masses of lymphoid tissue appear to make a stubborn effort to replace the excised organs after large tonsils and adenoids have been supposedly cleaned out surgically. Thus if an adenoidectomy is performed before the child is 3 years old, it is more than likely that a secondary adenoidectomy will be necessary.

Further, it is now recognized, that, in a certain number of patients with ear problems, the careful removal of the nasopharyngeal disease is actually more important than tonsillectomy.

\section{Orthodontic management:}

Various modalities of orthodontic management of upper airway obstruction are as follows:

1. Maxillary expansion- Expansion of maxilla improves nasal volume and nasal flow thus alleviating the symptoms of upper airway obstruction due to adenotonsillar hypertrophy. There are four techniques for expanding narrow maxillae: (a) orthodontic expansion e.g. by plate therapy or using a quad-helix appliance, (b) rapid maxillary expansion (RME) using a hyrax screw or palatal distractor, (c) surgically assisted orthodontic maxillary expansion and (d) transverse segmental osteotomy. ${ }^{22}$

It is relatively easy to widen the maxilla by opening the midpalatal suture before and during adolescence, but this becomes progressively more difficult as patients become older. $\mathrm{T} h$ e chances of successful opening of the suture are nearly $100 \%$ before age 15 , but begin to decline thereafter because of the increased 
interdigitation of the sutures. ${ }^{23}$ Thereafter combined orthodontic and surgical approach is required to avoid complications such as fractures of the alveolar process. ${ }^{23}$

2. Mandibular repositioning devices (MRDS) : The most common intervention for obstructive sleep apnea is removal of the adenoids and tonsils. This procedure is associated with the surgical risks in children with comorbidities and patients without hypertrophy of the lymphatic tissue. This is why oral orthodontic appliances (OA; or mandibular advancement device, MAD) are used to enlarge the upper airway and prevent its collapse by displacing the mandible forward.

For patients with mild-to-moderate OSA a removable oral appliance that repositions the mandible forward e.g. removable herbst appliance is a good option. The success of this type of treatment is based on a somewhat similar response of the tissues to that of orthognathic surgery. These devices have their effect because of the attachment of the mandible to the tongue, pharyngeal dilator muscles, and indirectly the soft palate. By moving the mandible forward, it brings these structures that make up the lumen of the oropharynx forward as well, thereby increasing the airway space. ${ }^{24,25}$

3. Planned bilateral sagittal split surgery advancing the mandible widens the airway and relieves the symptoms of upper airway obstruction.

\section{Conclusion}

While many studies document a clear relationship between airway obstruction and dentofacial development, some fail to demonstrate any such relationships. The evaluation of nasal airway patency is complicated; more so when the possibility exists that airways may clinically appear inadequate but is quite functional physiologically.

Whereas, too much emphasis has been placed in some studies on different methods to assess the nasopharyngeal airflow or the nasal resistance, individual's mandibular, tongue, and head posture response to nasal obstruction is far more important. These postural modifications can serve as precursors/indicators for need of surgical and/or orthodontic intervention to reduce the dentofacial abnormalities in cases of hypertrophied adenoid and tonsils.

Certain objective guidelines for the management of adenotonsillar hypertrpohy induced airway obstruction have been presented in the article above which can help solve the existing dilemma of when and when not to remove the adenotonsillar lymphatic tissue.

Surgical intervention, the most common treatment historically might not be the most optimal or the most desirable given the availability of alternates like orthodontic and/or pharmacological treatment options.

While, surgical intervention might be necessary in some patients, it should be used only when mandatory rather than the default intervention.

\section{References}

[1]. Aboudara C, Nielsen I, Huang JC, Maki K, Miller AJ, Hatcher D. Comparison of airway space with conventional lateral headfilms and 3-dimensional reconstruction from cone-beam computed tomography. Am J Orthod Dentofacial Orthop. 2009 Apr; 135(4):46879.

[2]. Souki MQ, Souki BQ, Franco LP, Becker HM, Araújo EA. Reliability of subjective, linear, ratio and area cephalometric measurements in assessing adenoid hypertrophy among different age groups. Angle Orthod. 2012 Nov; 82(6):1001-7.

[3]. Hellings P, Jorissen M, Ceuppens JL. The Waldeyer's ring. Acta Otorhinolaryngol Belg. 2000; 54(3):237-41.

[4]. Diamond O. Tonsils and adenoids: why the dilemma? Am J Orthod. 1980 Nov; 78 (5):495-503.

[5]. Linder-Aronson S, Woodside DG. Excess face height malocclusion: Etiology, diagnosis and treatment. $1^{\text {st }}$ ed. Quintessence Pub; 2000 .

[6]. The effects of enlarged adenoids on a developing malocclusion Dr.Kevin Williams ${ }^{1}$, Dr. Derek Mahony

[7]. Rubin RM. Effects of nasal airway obstruction on facial growth. Ear, Nose \& Throat J. 1987; 66:44-53.

[8]. HARTSOOK JT. Mouth breathing as a primary etiologic factor in the productionof malocclusion. J Dent Child. 1946; 13(4):91-4

[9]. Coelho AR, Tanaka O, Ribeiro JS, Machado MA, Camargo ES. Transverse craniofacial dimensions in Angle Class II, Division 1 malocclusion according to breathing mode. Braz Oral Res. 2010 Jan-Mar; 24(1):70-5.

[10]. Vig PS, Sarver DM, Hall DJ, et al. Quantitative evaluation of nasal airflow in relation to facial morphology. Am J Orthod 1981; 79:263-272.

[11]. Holmberg H, Linder-Aronson S. Cephalometric radiographs as a means ofevaluating the capacity of the nasal and nasopharyngeal airway. Am J Orthod. 1979 Nov; 76(5):479-90.

[12]. McNamara JA Jr. A method of cephalometric evaluation. Am J Orthod. 1984 Dec; 86(6):449-69.

[13]. Kemaloglu YK, Goksu N, Inal E, Akyildiz N. Rdiographic evaluation of children with nasopharyngeal obstruction due to the adenoid. Ann Otol Rhinol Laryngol. 1999 Jan; 108(1):67-72.

[14]. Oh KM, Kim MA, Youn JK, Cho HJ, Park YH. Three-dimensional evaluation of the relationship between nasopharyngeal airway shape and adenoid size in children. Korean J Orthod. 2013 Aug; 43(4):160-7.

[15]. Forum on the tonsil and adenoid problem in orthodontics. Questions and answers. Am J Orthod. 1968 Jul;54(7):508-1

[16]. Ricketts RM. Respiratory obstruction syndrome. Am J Orthod 1968; 54:495-514 Pereira SR, Bakor SF, Weckx LL.

[17]. Adenotonsillectomy in facial growing patients: spontaneous dental effects. Braz J Otorhinolaryngol. 2011 Sep-Oct;77(5):600-

[18]. McNamara JA. Influence of respiratory pattern on craniofacial growth. Angle Orthod. 1981 Oct; 51(4):269-300.

[19]. Cassano P, Gelardi M, Cassano M, Fiorella ML, Fiorella R. Adenoid tissuerhinopharyngeal obstruction grading based on fiberendoscopic findings: a novel approach to therapeutic management. Int J Pediatr Otorhinolaryngol. 2003 Dec; 67(12):1303-9.

[20]. Peterson J, Losek JD. Post-tonsillectomy hemorrhage and pediatric emergency care. Clin Pediatr (Phila). 2004 Jun; 43(5):445-8. 
[21]. Stuck BA, Götte K, Windfuhr JP, Genzwürker H, Schroten H, Tenenbaum T. Tonsillectomy in children. Dtsch Arztebl Int. 2008 Dec; 105(49):852-60.

[22]. Kiliç N, Oktay H. Effects of rapid maxillary expansion on nasal breathing and some naso-respiratory and breathing problems in growing children: a literature review. Int J Pediatr Otorhinolaryngol. 2008 Nov; 72(11):1595-601.

[23]. Proffit WR, Fields HW, Sarver DM. Contemporary Orthodontic. $4^{\text {th }}$ ed.Mosby; 2006

[24]. Effects of Mandibular Advancement Device (MAD) on Airway Dimensions Assessed With Cone-Beam Computed Tomography Jennifer A. Haskell, John McCrillis, Bruce S. Haskell, James P. Scheetz, William C. Scarfe, and Allan G. Farman Semin Orthod 2009;15:132-158.)

[25]. Stellzig-Eisenhauer A, Meyer-Marcotty P. Interaction between otorhinolaryngology and orthodontics: correlation between the nasopharyngeal airway and the craniofacial complex. GMS Curr Top Otorhinolaryngol Head Neck Surg. 2010; 9:Doc04. 